biblio.ugent.be

The UGent Institutional Repository is the electronic archiving and dissemination platform for all UGent research publications. Ghent University has implemented a mandate stipulating that all academic publications of UGent researchers should be deposited and archived in this repository. Except for items where current copyright restrictions apply, these papers are available in Open Access.

This item is the archived peer-reviewed author-version of:

Alignment-tolerant interfacing of a photonic integrated circuit using back side etched silicon microlenses

J. Missinne, N. Teigell Benéitez, N. Mangal, J. Zhang, A. Vasiliev, J. Van Campenhout, B. Snyder, G. Roelkens, and G. Van Steenberge

In: Proceedings of the SPIE, 10923, page 1092304 (7 pages), 2019.

https://doi.org/10.1117/12.2506159

To refer to or to cite this work, please use the citation to the published version:

J. Missinne, N. Teigell Benéitez, N. Mangal, J. Zhang, A. Vasiliev, J. Van Campenhout, B. Snyder, G. Roelkens, and G. Van Steenberge (2019). "Alignment-tolerant interfacing of a photonic integrated circuit using back side etched silicon microlenses." Proc. SPIE. 10923. 10.1117/12.2506159. 


\title{
Alignment-tolerant interfacing of a photonic integrated circuit using back side etched silicon microlenses
}

\author{
J. Missinne*,1, N. Teigell Benéitez ${ }^{2}$, N. Mangal ${ }^{1,3}$, J. Zhang $^{2}$, A. Vasiliev ${ }^{2}$, J. Van Campenhout $^{3}$, B. \\ Snyder $^{3}$, G. Roelkens ${ }^{2}$, G. Van Steenberge ${ }^{1}$ \\ ${ }^{1}$ Center for Microsystems Technology, Ghent University-imec, Technologiepark 126, B-9052 \\ Zwijnaarde, Belgium. \\ ${ }^{2}$ Photonics Research Group, Ghent University-imec, Technologiepark 126, B-9052 Zwijnaarde, \\ Belgium. \\ ${ }^{3}$ imec, Kapeldreef 75, B-3001 Leuven, Belgium.
}

\begin{abstract}
Directly interfacing a photonic integrated circuit allows at best an alignment tolerance of a few micrometer due to the small dimensions of optical (coupling) features on chip, but when using microlenses integrated on the substrate-side, alignment tolerances for interfacing the chips can greatly be relaxed. This is demonstrated on a $750 \mu \mathrm{m}$ thick chip with standard grating couplers (operation wavelength around $1550 \mathrm{~nm}$ ). Low roughness silicon microlenses were realized by transferring reflowed photoresist into the silicon substrate using reactive ion etching. The microlens allows interfacing the chip from the backside with an expanded beam, drastically increasing lateral alignment tolerances. A $1 \mathrm{~dB}$ alignment tolerance of $\pm 8 \mu \mathrm{m}$ and $\pm 11 \mu \mathrm{m}$ (along and perpendicular to the grating coupler direction, respectively) was experimentally found when a $40 \mu \mathrm{m}$ mode field diameter beam was used at the input.
\end{abstract}

Keywords: back side, photonic integrated circuit, silicon microlens, tolerant alignment

\section{INTRODUCTION}

Photonic Integrated Circuits (PICs) are commonly interfaced with single-mode fibers using grating couplers by performing active alignment [1]. However, due to the relatively small mode field diameter (MFD) of these fibers, their alignment with respect to the grating couplers is very critical (about $2 \mu \mathrm{m}$ for a $1 \mathrm{~dB}$ lateral alignment tolerance), making it difficult to perform passive alignment. Especially for disposable chips, such as for sensor applications, this is not ideal since an alignment step is required each time the photonic sensor chip is replaced. Furthermore, fibers interfaced via the top side (or device side) of the chip can block active sensor regions. Both of these issues issues can be resolved by interfacing the (sensor) chip from the back side using microlenses, as illustrated in Figure 1. The idea is to expand the beam emitted by the grating coupler through the bulk silicon substrate and then collimate it with the help of microlenses fabricated at the back side of the chip. An expanded mode field diameter of the resultant beam provides relaxed alignment tolerance of the chip with respect to the readout. This concept was shown before using hybrid integration of glass microlenses on the top side [2] or polymer microlenses on the back side [3] of a PIC. In the current paper, this concept is implemented using monolithically integrated microlenses on the back of regular, shallow-etched grating couplers.
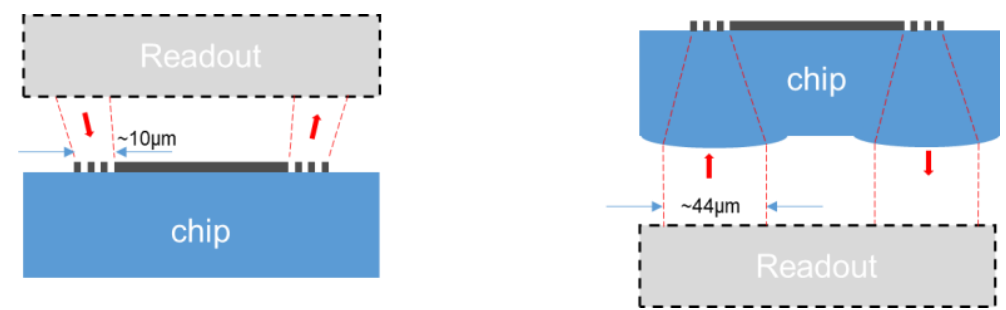

Figure 1. Conventional method for interfacing grating couplers on a PIC (left) and proposed method using integrated microlenses.

*jeroen.missinne@ugent.be; phone +32 9264 5621; fax +329264 3594; www.cmst.be 


\section{OPTICAL COUPLING CONCEPT}

A regular grating coupler, designed for a peak wavelength at $1550 \mathrm{~nm}$, emits a beam that is mode-matched with a singlemode fiber with a spot diameter of $10.4 \mu \mathrm{m}$. Furthermore, a regular grating emits about $30 \%(5 \mathrm{~dB})$ of in the input power into each of the upward and downward direction respectively [1]. The beam emitted in the downward direction expands while traveling through the substrate approximately dictated by the gaussian beam theory (approximately since the emitted beam is usually not perfectly Gaussian). If a suitable lens is then placed on the chip backside, this divergent beam can be collimated. Conversely, a collimated beam from an external source with the correct diameter, which is launched onto the lens, can be focussed into a $10 \mu \mathrm{m}$ spot on the grating coupler. When this larger, expanded beam at the microlens side is used for interfacing the chip, the lateral alignment tolerances are greatly relaxed, compared to directly coupling from a single mode fiber to the grating coupler.

\subsection{Lens design}

The required lens radius of curvature (ROC) can be estimated from the formula for refraction at a spherical silicon-air surface (geometric optics approximation), i.e. ROC $\approx f\left(\left(n_{s i}-n_{\text {air }}\right) / n_{s i}\right)$, where $f$ is the lens focal distance and $n_{s i}$ and $n_{\text {air }}$ are the refractive index (at $\lambda=1550 \mathrm{~nm}$ ) of silicon and air respectively. To collimate the divergent beam emitted by the grating coupler, the focal distance of the lens should be the same as the distance between grating coupler and lens vertex $(\sim 750 \mu \mathrm{m})$, requiring an estimated ROC of $546 \mu \mathrm{m}$.

The lens design was further fine-tuned for maximum collimation using the Zemax OpticStudio which allows both ray tracing and gaussian beam propagation simulations (using the physical optics module). The resulting design, showing the required ROC of $568 \mu \mathrm{m}$, is visualized in Figure 2. The diameter of the beam is defined by the wavelength (design wavelength $=1550 \mathrm{~nm}$ ), refractive index and thickness of the silicon substrate and by the numerical aperture of the grating coupler (in case of the ray tracing model) or by the waist of the beam emitted by the grating coupler (in case of the Gaussian beam approximation). Taking these into acount, the diameter of the beam at the microlens will be $44 \mu \mathrm{m}$ for a standard grating coupler (waist $5 \mu \mathrm{m}, 10^{\circ}$ coupling angle in air) on a chip with a substrate thickness of $750 \mu \mathrm{m}$.

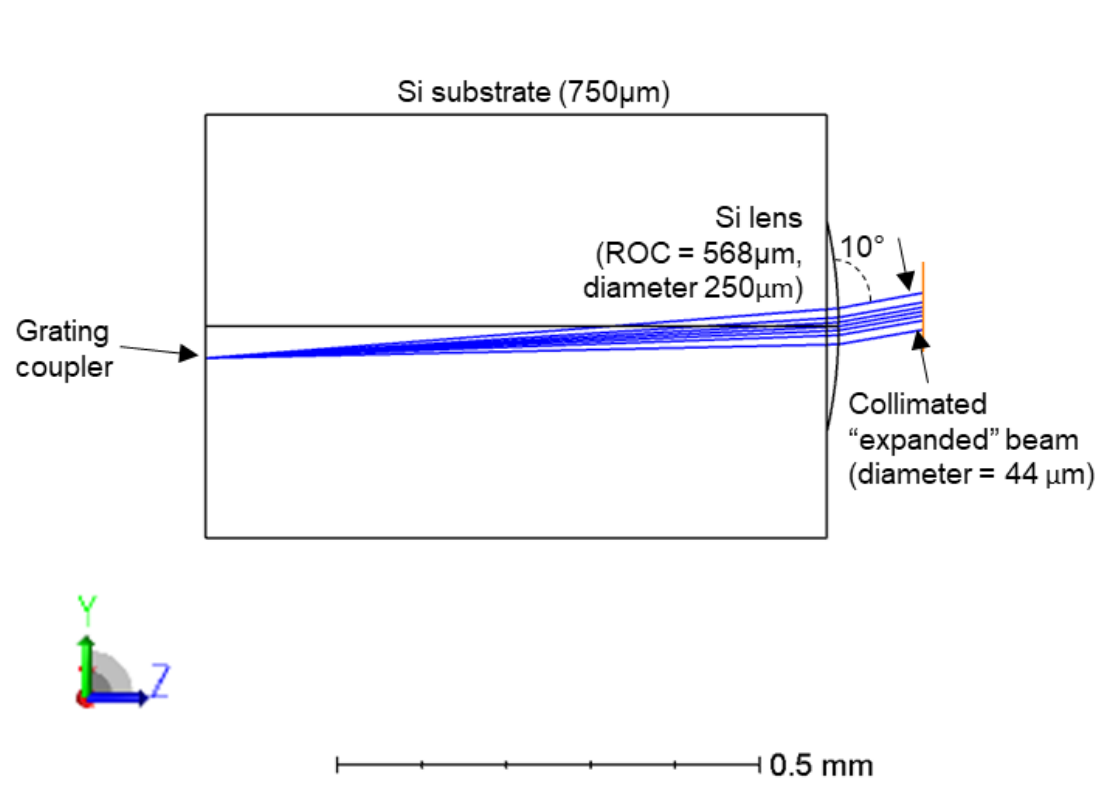

Figure 2. Zemax ray-trace model for the monolithic integration of a microlens on the backside of a photonic chip, including the relevant parameters (ROC: radius of curvature).

Fabricating microlenses using the reflow technique as described below is prone to certain process tolerances. Therefore, the influence of variations of the lens ROC on the coupling effiency was studied using a Gaussian beam approximation model. For this study, the grating coupler was assumed to emit a Gaussian beam with a $5 \mu \mathrm{m}$ waist $(10 \mu \mathrm{m}$ spot size $)$ which propagates through the silicon substrate and is eventually collimated by the microlens. The normalized coupling efficiency was determined by calculating the overlap integral between the collimated beam and a Gaussian mode with a 
$44 \mu \mathrm{m}$ diameter, positioned $100 \mu \mathrm{m}$ away from the microlens (relative coupling efficiency equal to 1 in case the lens ROC is exactly as designed). Figure 3 shows that there is a relatively large tolerance on the ROC of the microlens, i.e. a $1-\mathrm{dB}$ drop in coupling efficiency occurs for a deviation of $+/-130 \mathrm{um}$ in the radius of curvature of the microlens. Of course, when the ROC deviates from the design, the beam will not be perfectly collimated, which would impact its positioning tolerance in the z-direction (direction along the beam, away from the lens).

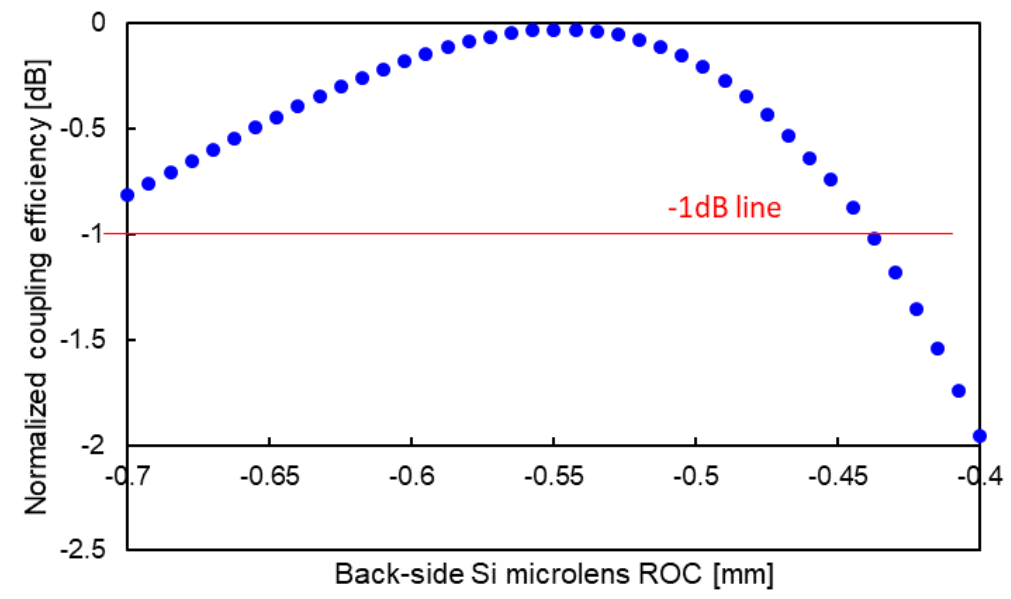

Figure 3. Influence of the lens ROC on the normalized coupling efficiency.

\subsection{Lateral alignment tolerances}

The lateral alignment tolerance of the PIC with back side microlens is estimated by calculating the change in coupling efficiency when an external Gaussian beam (beam diameter $44 \mu \mathrm{m}, 100 \mu \mathrm{m}$ away from the microlens) is displaced in the $\mathrm{x}$ - and $\mathrm{y}$-direction above the microlens. Again, the coupling efficiency is estimated by calculating the overlap integral with a Gaussian mode with spot size $10 \mu \mathrm{m}$ at the location of the grating coupler. From Figure 4 it can be seen that the $1 \mathrm{~dB}$ alignment tolerance is $\pm 10 \mu \mathrm{m}$ in that case. This tolerance is the same for both directions which results from the assumption that the grating coupler diffracts a circular Gaussian beam. Since this is only an approximation and since a grating coupler is wavelength selective, the actual alignment tolerance will slightly differ in $\mathrm{x}$ - and $\mathrm{y}$-direction, as discussed in section 4 .

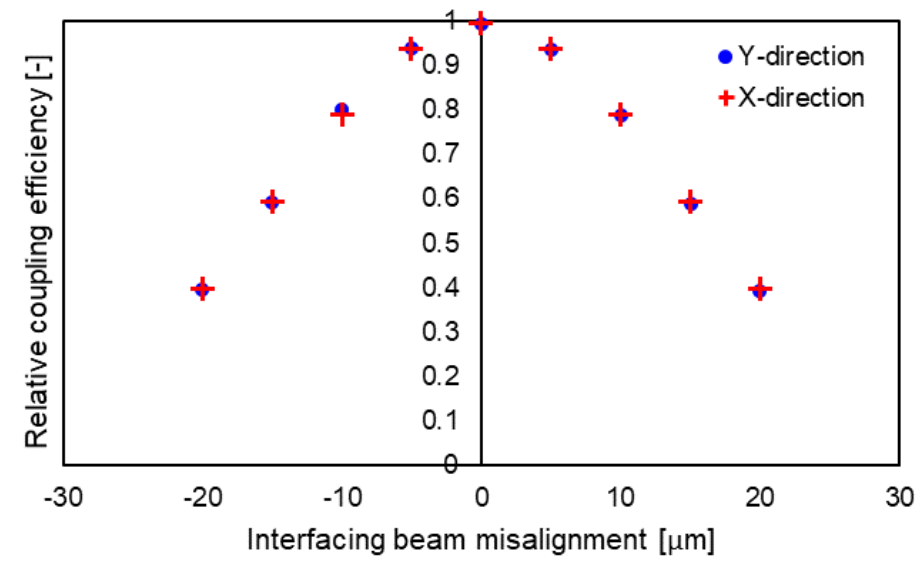

Figure 4. Influence of the interfacing beam misalignment on the relative coupling efficiency. 


\section{SILICON MICROLENS FABRICATION}

The microlenses are fabricated by reflowing AZ 4562 resist [4] features on the back side of the PIC and subsequently transfering them into the silicon substrate using reactive ion etching (RIE). During this process the device side was protected with a $10 \mu \mathrm{m}$ thick AZ 4562 resist layer.

This process is schematically illustrated in Figure 5. First, the rough backside of the PIC was polished to make it optically smooth. Then, disks were patterned using lithography and aligned with respect to the grating couplers on the device side using an EVG 602 double side mask aligner. Afterwards, the PIC was heated leading to reflow of the resist structures yielding spherical lens surfaces. The ROC of these lenses depends on the diameter and thickness of the initial disks. For achieving the required lens with an ROC equal to $568 \mu \mathrm{m}$, a $250 \mu \mathrm{m}$ diameter disk with a $11.5 \mu \mathrm{m}$ thickness was required.
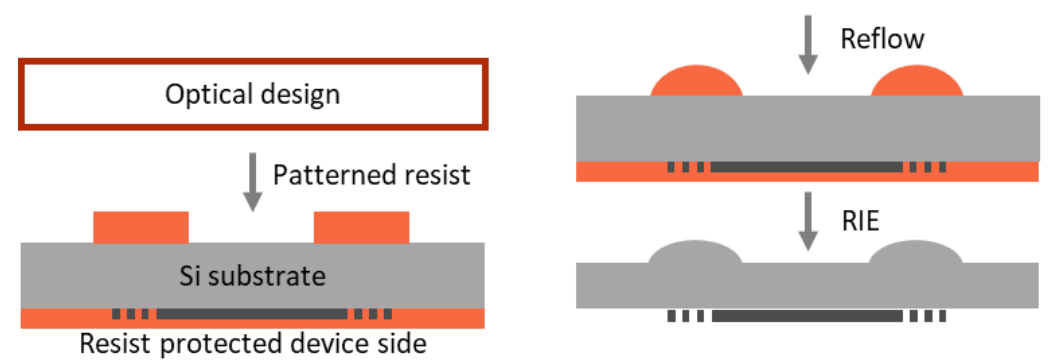

Figure 5. Schematic overview of the microlens fabrication process.

Once the resist lenses were realized, they were transferred into the silicon substrate using an RIE process optimized for achieving a 1:1 resist:silicon etch ratio (Oxford RIE-ICP System 100, 40 mtorr, $20^{\circ} \mathrm{C}, 75 \mathrm{~W}, \mathrm{SF}_{6} 15.2 \mathrm{sccm}, \mathrm{O}_{2}$ $38 \mathrm{sccm}, \mathrm{t}=180 \mathrm{~min}$ ). This way, the etch process does not change the ROC as defined by the photoresist structure.

The surface profiles of the resulting silicon micolenses were measured using a mechanical stylus-based profiler (Veeco Dektak), as shown in Figure 6. An ROC of $602 \mu \mathrm{m}$ was found (calculated by performing a circle fitting through the measured data), which was slightly larger than the targetted ROC, but still well within the $1 \mathrm{~dB}$ tolerance range as discussed above and illustrated in Figure 3. This could be due to process variations, arising mainly in the resist thickness and the etch ratio. Figure 6 also shows some defects on the etched lens, which were likely caused by the contamination of the chamber of the RIE tool.

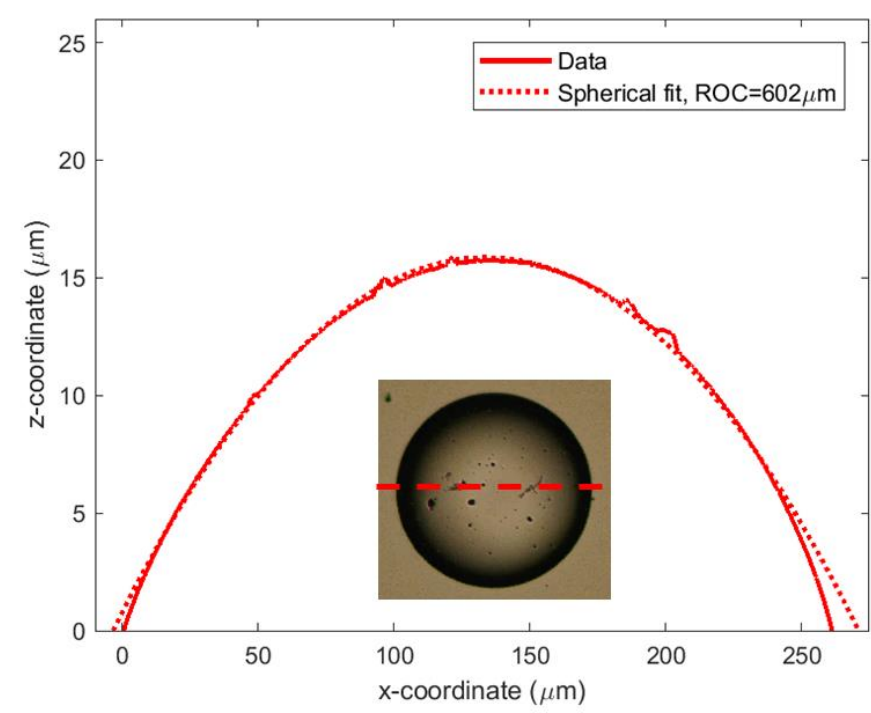

Figure 6. Cross-sectional profile of a silicon microlens with a $\sim 250 \mu \mathrm{m}$ diameter etched into the backside of a PIC. The inset shows an image of the same microlens indicating where the cross-sectional profile was recorded (dashed red line). 
The surface roughness of the silicon microlenses post etching was measured using an optical profiler (Veeco Wyko). After subtracting the desired spherical lens shape from the measured profile, a root-mean-square roughness below $7 \mathrm{~nm}$ was found, determined over the central 50 x $50 \mu \mathrm{m}^{2}$ lens area.

\section{RESULTS: LATERAL ALIGNMENT TOLERANCES}

\subsection{Setup}

The alignment tolerance of the PIC with respect to an external readout system was assessed by launching a $40 \mu \mathrm{m}$ diameter beam onto the input microlens using a thermally expanded core (TEC) SMF-28 single mode fiber, as illustrated in Figure 7. At the same time, a $50 \mu \mathrm{m}$ diameter core multimode fiber was used as a collecting mechanism at the output side. Both fibers were placed as close to the microlens as possible (visually, using a camera system). A multimode fiber with large numerical aperture was chosen so that the coupling tolerances at the input side could be studied without needing to reposition the output fiber. This is an approximation for the situation in which a relatively large detector is used to inferface the output grating coupler.

The output multimode fiber was kept fixed while the TEC fiber was scanned over the input microlens in $\mathrm{x}$ - and $\mathrm{y}$ direction in steps of $5 \mu \mathrm{m}$. At each point $(\mathrm{x}, \mathrm{y})$, the coupling efficiency was recorded for wavelengths between $1500 \mathrm{~nm}$ and $1620 \mathrm{~nm}$ using a tunable diode laser at the input (TE polarization) and photodiode at the output. The recorded signals were referenced to the power measured when directly connecting the tunable laser with the photodiode using a patchcord.

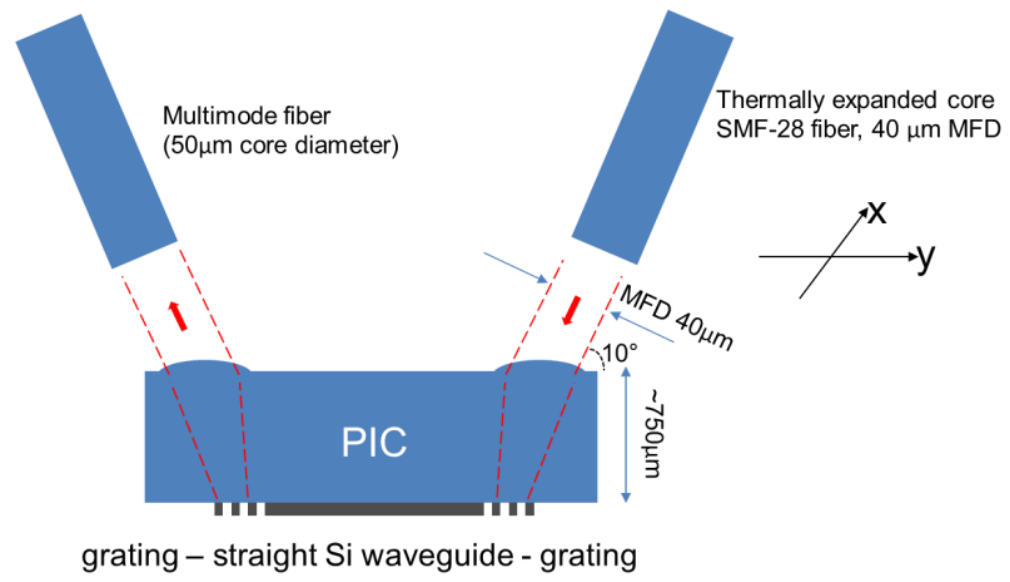

Figure 7. Test configuration for measuring the alignment tolerances on the incoupling side of the PIC.

\subsection{Results}

Figure 8 shows the measured insertion loss spectra at several $\mathrm{x}$ - and $\mathrm{y}$-positions of the input fiber. When scanning the fiber in the y-direction (i.e. the longitudinal direction along the waveguide), it can be seen that the peak coupling efficiency experiences a blue-shift. This can be understood as follows. The microlens is designed to focus the collimated input beam onto its focal point, i.e. on the grating coupler, regardless of its (x,y)-position (approximately). This means, however, that the angle of incidence on the grating coupler will change as a function of the y-position, which is know to cause a shift in the insertion loss spectrum. Likewise, when scanning the fiber in the $\mathrm{x}$-direction over the microlens, the incoupling angle in a direction transverse to the grating will change, leading to a reduction in coupling efficiency without spectral shift.

The positioning tolerance of the input beam can therefore best be determined at a fixed operation wavelength. Here, an operation wavelength $\lambda=1560 \mathrm{~nm}$ was chosen since this wavelength yielded the maximum coupling efficiency at perfect alignment. As such, the insertion loss was determined as a function of input fiber position, in $\mathrm{x}$ - and $\mathrm{y}$-direction, see Figure 9. From this plot, a $1 \mathrm{~dB}$ alignment tolerance of $\pm 11 \mu \mathrm{m}$ and $\pm 8 \mu \mathrm{m}$ was found in the $\mathrm{x}$ - and $\mathrm{y}$-direction respectively. This is about 5 times larger than the case in which a grating coupler is directly interfaced with a single mode fiber. 
Note that the overall peak coupling efficiency $(-26 \mathrm{~dB})$ is limited by the fact that in-house available chips with regular grating couplers were used which were not optimized for backside coupling. Furthermore, no anti-reflection coating was applied on the microlenses at this moment, leading to additional Fresnell losses. This back side coupling efficiency of a single grating was estimated by thinning a separate, but identical chip to $100 \mu \mathrm{m}$, applying a $200 \mathrm{~nm} \mathrm{Si} \mathrm{N}_{4}$ anti-reflection coating and interfacing both grating couplers from the back side with SMF-28 single mode fibers. As such, a $-11 \mathrm{~dB}$ coupling loss per grating coupler was measured, explaining a large part of the total insertion loss.
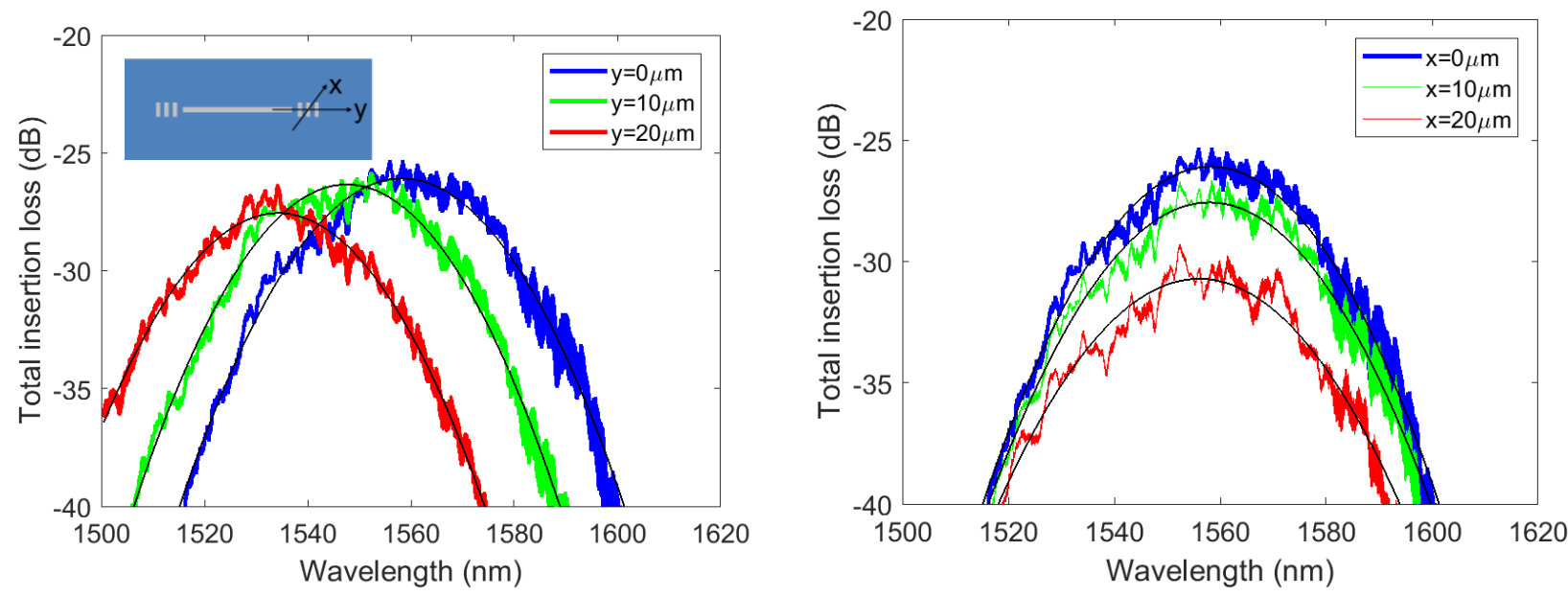

Figure 8. Evolution in the insertion loss spectra for sweeping the input fiber in the y-direction (left) and x-direction (right).

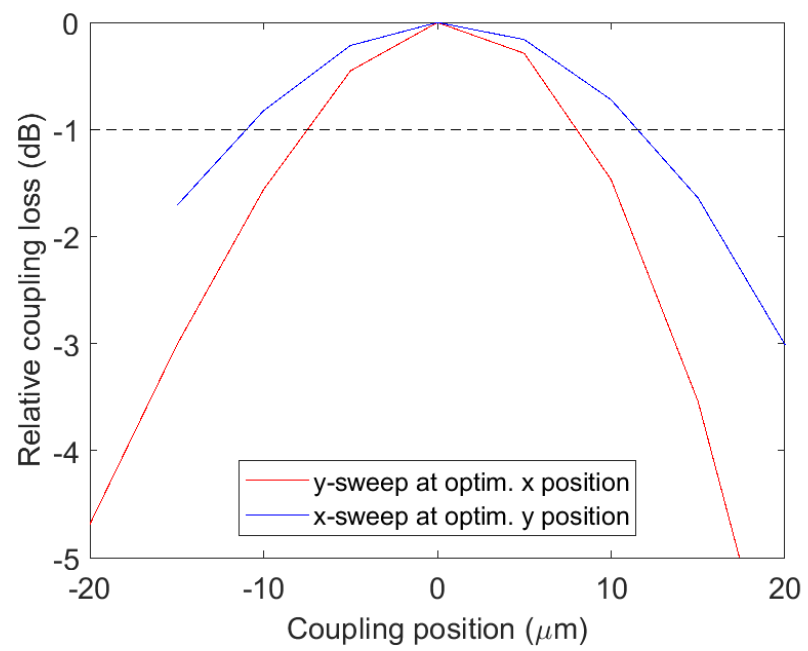

Figure 9. Relative coupling loss as a function of input fiber position in $\mathrm{x}$ - and $\mathrm{y}$-direction, from which the $1 \mathrm{~dB}$ alignment tolerance can be extracted.

\section{CONCLUSIONS}

A concept for relaxing the alignment tolerances of a photonic integrated circuit (with grating couplers) with respect to a readout system was presented. Using microlenses monolithically integrated on the chip backside, a $1 \mathrm{~dB}$ alignment tolerance of $\pm 11 \mu \mathrm{m}$ and $\pm 8 \mu \mathrm{m}$ in the direction along and across the grating coupler was found respectively. The overall peak coupling efficiency can be improved by optimizing the grating couplers for downward directionality, by applying an anti-reflection coating on the microlenses and by further fine-tuning of the etching process. The concept is especially useful for sensor applications, since the chips can more easily be replaced without the need for very accurate alignment. 


\section{ACKNOWLEDGEMENT}

The AQUARIUS project has received funding from the European Union's Horizon 2020 research and innovation programme under grant agreement No. 731465.

This project is an initiative of the Photonics Public Private Partnership.

\section{REFERENCES}

[1] D. Taillaert, W. Bogaerts, P. Bienstman, T. F. Krauss, P. V. Daele, I. Moerman, S. Verstuyft, K. D. Mesel, and R. Baets, "An out-of-plane grating coupler for efficient butt-coupling between compact planar waveguides and single-mode fibers," IEEE Journal of Quantum Electronics, 38(7), 949-955 (2002).

[2] C. Scarcella, K. Gradkowski, L. Carroll, J. Lee, M. Duperron, D. Fowler, and P. O'Brien, "Pluggable SingleMode Fiber-Array-to-PIC Coupling Using Micro-Lenses,” IEEE Photonics Technology Letters, 29(22), 19431946 (2017).

[3] N. Mangal, J. Missinne, G. Roelkens, J. Van Campenhout, G. Van Steenberge, and B. Snyder, "ExpandedBeam Through-Substrate Coupling Interface for Alignment Tolerant Packaging of Silicon Photonics," OSA Technical Digest (online). Tu2A.1.

[4] Microchemicals, "AZ4562 photoresist microchemicals," https://www.microchemicals.com/products/photoresists/az_4562.html (19 December 2018). 